\title{
Magneto-optical imaging of flux penetration into arrays of $\mathrm{Bi}_{2} \mathrm{Sr}_{2} \mathrm{CaCu}_{2} \mathrm{O}_{8}$ microdisks
}

\author{
M. R. Connolly, ${ }^{1}$ M. V. Milošević, ${ }^{1,2}$ S. J. Bending, ${ }^{1}$ and T. Tamegai ${ }^{3}$ \\ ${ }^{1}$ Department of Physics, University of Bath, Claverton Down, Bath BA2 7AY, United Kingdom \\ ${ }^{2}$ Departement Fysica, Universiteit Antwerpen, Groenenborgerlaan 171, B-2020 Antwerpen, Belgium \\ ${ }^{3}$ Department of Applied Physics, The University of Tokyo, Hongo, Bunkyo-ku, Tokyo 113-8656, Japan
}

(Received 14 July 2008; published 1 October 2008)

\begin{abstract}
We have used differential magneto-optical (MO) imaging to investigate the mixed state of superconducting $\mathrm{Bi}_{2} \mathrm{Sr}_{2} \mathrm{CaCu}_{2} \mathrm{O}_{8+\delta}$ (BSCCO) microdisks fabricated on a single-crystal sample. MO difference images of the stray field distribution over a range of out-of-plane fields allow us to distinguish between flux that is penetrating the disks and that entering the underlying BSCCO platelet. We find that flux preferentially flows along linear defects into the interstitial platelet regions up to a characteristic field $H_{p}$, above which flux enters the disks. We identify this as the field of first penetration of pancake vortices over the Bean-Livingston barrier around the disks, where $H_{p}(T)$ at intermediate temperatures is well described by an exponentially decaying function with a characteristic temperature $T_{0}=19 \mathrm{~K}$. At a given temperature, a minority of the disks exhibit a lower penetration field and we correlate the location of these disks with the linear defects in the BSCCO crystal.
\end{abstract}

DOI: 10.1103/PhysRevB.78.132501

PACS number(s): 74.78.-w, 74.25.Ha, 74.25.Op, 74.72.Hs

Bulk type-II superconductors in a magnetic field exhibit a flux-free state which survives to a lower critical field $H_{c 1}$. Above this field superconductivity is partially destroyed by the nucleation of flux-quantized vortices at lateral surfaces and their subsequent penetration into the body of the sample. An important consequence of the distortion of the edge (surface) currents during vortex nucleation is the appearance of a local attraction between the vortex and the surface. This attraction can be viewed as arising from an external image antivortex, whose presence ensures that there is no net current perpendicular to the surface. The image antivortex effectively delays the transition from the flux-free to the vortex state until the applied field reaches a penetration field $H_{p}$ $>H_{c 1}$, and the potential barrier which must be surmounted to enter the sample is called the Bean-Livingston barrier (BLb). ${ }^{1}$

This scenario is complicated by the fact that vortices can be thermally excited over the BLb at finite temperatures. Hence, provided sufficient time elapses after application of the field, the transition will occur close to $H_{c 1}$, as for an infinite (bulk) sample. ${ }^{2}$ For finite measurement times, however, the magnitude and temperature dependences of the penetration field differ dramatically from $H_{c 1}$ and several studies have attempted to determine the form of $H_{p}(T)$, particularly in the layered cuprate superconductor $\mathrm{Bi}_{2} \mathrm{Sr}_{2} \mathrm{CaCu}_{2} \mathrm{O}_{8}$ (BSCCO). ${ }^{3,4}$

With the applied field along the high-symmetry $c$ axis, the highly anisotropic electronic structure of BSCCO is reflected in the decomposition of flux lines into stacks of weakly coupled "pancake vortices" (PVs) whose currents are confined to the $\mathrm{CuO}_{2}$ planes. ${ }^{5}$ Since the PVs are only weakly coupled to one another, an individual PV can surmount the BLb independently of the rest of the stack, ${ }^{6,7}$ which is then subsequently dragged after it. It is now well established that the thermal excitation of individual PVs over the BLb is the rate-limiting step for flux penetration into BSCCO. For a layered sample with a pristine surface, it can be shown that $H_{p}$ is described by ${ }^{6}$

$$
H_{p}(T)=\gamma \frac{\sqrt{2} \kappa}{\ln \kappa} H_{c 1}(0)\left[1-\left(\frac{T}{T_{c}}\right)^{2}\right] e^{-T / T_{0}},
$$

where $\gamma$ is the scaling factor between the applied field and the local field at the edge of the sample, $T_{0}$ is a characteristic temperature, and $H_{c 1}(0)$ is the lower critical field at $T$ $=0 \mathrm{~K}$. While $\gamma$ corresponds to the demagnetization factor in a sample with ellipsoidal cross section, it is not straightforward to specify its value in a sample with arbitrary cross section. Indeed, experimental validation of Eq. (1) in real type-II samples is complicated by this, as well as the effects of bulk pinning and the edge shape or geometrical surface barrier $(\mathrm{Gb}){ }^{8}$ which together mask the true influence of the $\mathrm{BLb}$ on penetration. A common method for partially eliminating these other barriers is to modify the sample cross section, ${ }^{3,9}$ and there is good evidence to suggest that the BLb dominates the transition to the vortex state in BSCCO platelets at intermediate temperatures $(15 \mathrm{~K}<T<70 \mathrm{~K})$, while the $\mathrm{Gb}$ or bulk pinning dominates at higher temperatures $(T>70 \mathrm{~K}),{ }^{3,4}$ Due to the much larger energies required to surmount the $\mathrm{Gb}$ the probability of thermal excitation is also vanishingly small. ${ }^{8}$ Thus a measurement of the temperature dependence of the applied field at penetration is a conclusive way of distinguishing between the $\mathrm{Gb}$ and the BLb.

BSCCO microdisks were recently suggested ${ }^{4}$ as promising systems for studies of surface barriers in BSCCO due to their low bulk pinning barriers. However, a feature of BSCCO single crystals (and most other superconductors that are used to fabricate disks) is the presence of quenched microscopic disorder which inevitably locally perturbs the BLb. In BSCCO disorder commonly takes the form of linear defects (LDs) aligned close to the $a$-axis growth direction. While the origin and composition of these defects are currently unclear, evidence for their oxygen deficient stoichiometry, obtained by high-resolution scanning transmission electron microscopy-energy dispersive spectroscopy (STEM-EDS), ${ }^{10}$ is consistent with the observed enhanced pinning and preferential penetration of flux along the 


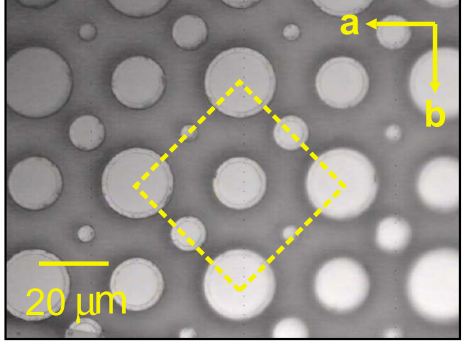

FIG. 1. (Color online) Optical micrograph of the disk array showing the orientation of the $40 \mu \mathrm{m} \times 40 \mu \mathrm{m}$ square unit cell (dashed outline) and the directions of the crystallographic $a$ and $b$ axes.

defects. ${ }^{11}$ The latter behavior is caused by the local suppression of superconductivity and is similar to that along weak intergranular links such as twin boundaries. Magneto-optical images of single crystals grown using the traveling solvent floating zone technique reveal that LDs extend along the entire length of the crystal with a nonuniform spacing, ${ }^{12}$ suggesting that they could be small-angle grain boundaries between individual crystallites. ${ }^{13}$ However, recent observations of a second drop in the in-plane resistivity at $T \approx 106 \mathrm{~K}$ support the idea that LDs are needlelike intergrowths of the higher $T_{c}$ Bi-2223 phase. ${ }^{14}$

In order to observe the interplay between a controlled sample geometry, quenched disorder, and the BLb, we have fabricated arrays of microdisks on the surface of a BSCCO single crystal and systematically observed the flux distribution as a function of sample history using differential magneto-optical imaging (MOI). We monitored the behavior of the threshold field $H_{p}(T)$ for penetration into the disks and compared this with known models for the BLb [cf., Eq. (1)]. As a further objective, we attempted to establish a link between the observed penetration field and position of the disks with respect to the linear defects in the crystal, thereby determining the local influence of quenched disorder on the BLb.

An as-grown BSCCO single crystal $\left(T_{c} \approx 91 \mathrm{~K}\right.$, dimensions $\approx 2.5 \mathrm{~mm} \times 2 \mathrm{~mm} \times 100 \mu \mathrm{m}$ ) was cleaved and arrays of microdisks were patterned into the surface to a depth of $d=300 \mathrm{~nm}$ using photolithography and argon-ion beam milling. A typical region of the array, shown in Fig. 1, consists of four disks with nominal diameters of 5, 10, 15, and $20 \mu \mathrm{m}$ arranged on the corners of a $20 \mu \mathrm{m} \times 20 \mu \mathrm{m}$ square cell with lattice constants inclined at approximately $45^{\circ}$ to the crystallographic $a$ axis of the crystal. The patterned area extended over 1-2 $\mathrm{mm}^{2}$ and was attached to a silicon substrate for mechanical stability.

The disk array was cooled below $T_{c}$ and imaged using a low-temperature magneto-optical (MO) system based on a cryocooler (details of the system can be found in Ref. 15). A Bi:yttrium iron garnet film was mechanically pressed onto the surface of the array, and the sample was fixed to a copper cold head. Optical access to the sample was achieved through a window on the top of the sample chamber. The angle between the analyzer and polarizer was slightly shifted from the point where the light reflected from the surface was extinguished (crossed polarizers) and a charge coupled de-

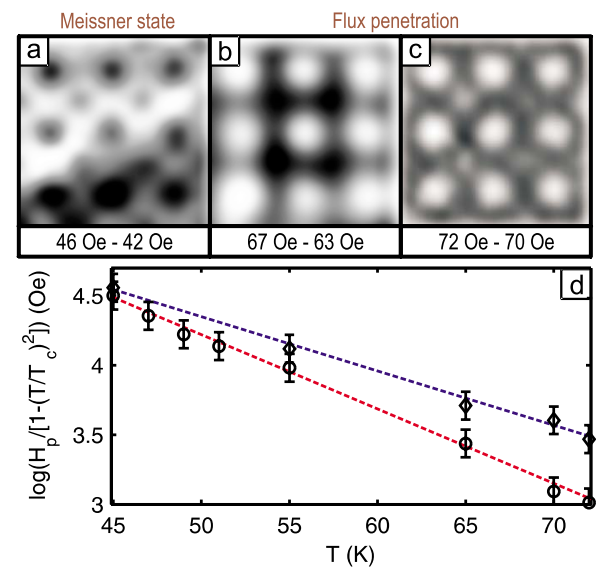

FIG. 2. (Color online) MO difference images (100 $\mu \mathrm{m}$ $\times 100 \mu \mathrm{m}$ ) at $T=45 \mathrm{~K}$ : (a) below $H_{p}$ (disks in Meissner state), (b) above $H_{p}$ for the $20 \mu \mathrm{m}$ disks, and (c) above $H_{p}$ for the $15 \mu \mathrm{m}$ disks. (d) Plot of $\log \left(H_{p} /\left[1-\left(T / T_{c}\right)^{2}\right]\right)$ vs $T$ of the experimental data for the $15 \mu \mathrm{m}(\diamond)$ and $20 \mu \mathrm{m}(\bigcirc)$ diameter disks and the theoretical fit (dashed lines) using Eq. (1).

vice (CCD) camera was then attached to the microscope. An out-of-plane field $H_{z}$ was applied using a copper coil wound directly around the sample space and powered using a manually controlled power supply. After the field was applied, the exposure time of the CCD was set to between 50 and $100 \mathrm{~ms}$ and the image averaged between 300 and 500 times, depending on the image quality and contrast. $H_{z}$ was then set to a new value and the measurement repeated, with each image taking approximately $10 \mathrm{~min}$ to acquire and process. The angle between the polarizer and analyzer was fine tuned using an electronically controlled stepper motor to find the strongest contrast in "difference" images, i.e., images obtained by subtracting two raw images captured under different applied fields. We exclusively use difference images in this study as they enable one to discern much subtler changes in the flux distribution by removing features unrelated to the influence of the applied field on the sample, such as domain patterns in the garnet.

Although the disks in our system are actually sitting on top of a BSCCO platelet we argue that this plays little role for the penetration of flux into the disks themselves since bulk vortex pinning will be small at our measurement temperature. Hence, once vortices enter into the platelet, they arrange themselves with distributions of $\mathbf{B}$ and $\mathbf{H}$ such that to good approximation $\mathbf{J}=$ curl $\mathbf{H}=0$, just as in free space. Prior to penetration the BLb at the sides of the disks presents a barrier to the entry of vortices into the disk. The screening currents are large on the top of the disk, pulling the tops of the vortices toward the center of the disk, but the vortex line tension balances this force. Even though there is no "surface" at the bottom of the disk, there must also be an effective sheet current similar to that on the top surface arising from $\mathbf{J}=$ curl $\mathbf{H}$ and the discontinuity in $\mathbf{H}$ between the vortexfilled region, where $H_{z} \approx H_{c 1}$, and the vortex-free region inside the disk, where $H_{z}=0$.

Figure 2 shows three MO difference images taken in increasing applied fields after cooling the sample to $T=45 \mathrm{~K}$. As anticipated, the change in the distribution of flux for the 



FIG. 3. (Color online) MO difference images $(0.4 \mathrm{~mm}$ $\times 0.3 \mathrm{~mm}, T=72 \mathrm{~K})$ constructed by subtracting the reference state in zero field from the images captured at applied fields of (a) 7.5 Oe and (b) 11.6 Oe. Arrows indicate the location of linear flux channels and the dashed outline in (a) indicates the unit cell of the array with corners on the $20 \mu \mathrm{m}$ disks (cf. Fig. 1).

low fields in Fig. 2(a) is strongly modulated by the disks. In particular, the intensity in the interstitial regions increases while the intensity over the unpenetrated disks remains low in comparison. Figures 2(b) and 2(c) show the difference between two slightly higher fields, where the opposite behavior is observed and the disks exhibit the strongest positive change in flux. Clearly, in Fig. 2(b) this is only happening in the $20 \mu \mathrm{m}$ disks, and it is not until a somewhat higher field that the smaller disks begin to be penetrated [see Fig. 2(c)]. The smallest disks could not be analyzed further in great detail due to the low spatial resolution in this experiment, but by systematically analyzing difference images at various temperatures we were able to establish the temperature dependence of the penetration field $H_{p}(T)$ when flux first enters the 20 and $15 \mu \mathrm{m}$ disks. These data are plotted in Fig. 2(d). The amount of flux entering the disks at penetration was too small to be measured for $T>80 \mathrm{~K}$, so there are no values close to the critical temperature $\left(T_{c} \approx 91 \mathrm{~K}\right)$. Note that $T_{c}$ itself was measured by finding the temperature at which contrast was lost at the edge of the platelet, and $H_{p}$ should obviously be zero at $T_{c}$.

The experimental form of $H_{p}(T)$ shows a rapid decay with increasing temperature. Curves obeying Eq. (1) were fitted to the data sets and the result is shown as a dashed line in Fig. 2(d), yielding values for $T_{0}$ of 19 and $25 \mathrm{~K}$ for the 20 and $15 \mu \mathrm{m}$ disks, respectively. The fit to the model yield a prefactor $\left[\gamma \sqrt{2} \kappa / \ln \kappa H_{c 1}(0)\right]$ of 990 Oe and a correlation coefficient of more than 0.98 , indicating excellent agreement. This result is consistent with previous studies, although we should emphasize that our fits are not sensitive to the behavior of $H_{p}(T)$ close to $T_{c}$, where the parabolic decrease in $H_{c}(T)$ begins to dominate. The values for $T_{0}$ are close to those obtained previously for disks $(35 \mathrm{~K}),{ }^{4}$ whiskers $(18 \mathrm{~K}),{ }^{16}$ and single crystals $(15 \mathrm{~K}){ }^{7}$ Using the most reliable data set for the $20 \mu \mathrm{m}$ disks we can estimate upper and lower bounds
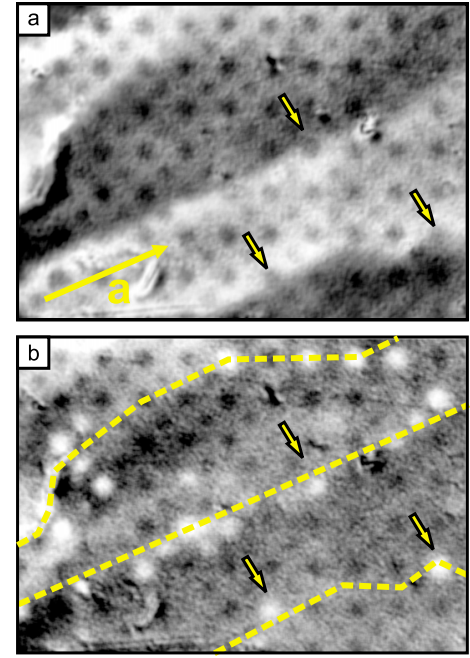

FIG. 4. (Color online) MO difference images $(0.4 \mathrm{~mm}$ $\times 0.3 \mathrm{~mm}, T=45 \mathrm{~K}$ ): (a) 32-27 Oe and (b) 50-49 Oe. Arrows in both images indicate the location of disks lying at the interface between the regions of differing vortex mobility. The dashed lines in (b) trace the edge of the channels observed in (a).

for $\gamma$ from the exponential prefactor. Taking $\kappa \approx 100 \pm 10$ (cf. Ref. 2) and $H_{c 1}(0 \approx 100 \pm 20$ Oe) we obtain $0.24<\gamma$ $<0.42$. This value is much closer to the expected value for a strip of width $W$ (taken equal to the diameter of our disks) with rectangular cross section, for which $\gamma=\sqrt{2 d / W} \approx 0.173$, than to one for a strip with an ellipsoidal cross section, for which $\gamma$ is an order of magnitude smaller $\gamma=d / W \approx 0.015 .{ }^{17}$

Having established the dominance of the BLb in the disks, we now discuss our observations relating to the inhomogeneous penetration of vortices into the underlying platelet. Figure 3 shows two $72 \mathrm{~K} \mathrm{MO}$ difference images captured at the two different indicated applied fields. Upon applying a small field of $H_{z}=7.5$ Oe [Fig. 3(a)], well defined linear regions of high-flux density, aligned close to the crystallographic $a$ axis, develop across the entire surface. This shows that, in line with previous studies, pancake vortices preferentially penetrate and accumulate along linear defects which are aligned close to the $a$ axis growth direction. ${ }^{18,19}$ The disks are still resolved in these regions due to their slightly lower MO intensity. A surface step, produced when the sample was cleaved, is visible in the lower left-hand corner, and the flow of the flux within the linear channels (indicated by arrows in Fig. 3) can be seen to terminate at the surface step. It is not until a higher field, $H_{z}=11.6 \mathrm{Oe}$, is applied [Fig. 3(b)] that the vortices in the linear regions "spill over" into the interstitial regions on the raised side of the step, where they continue to be guided by the linear channels, confirming their correlation down through the $c$ axis (perpendicular to the sample surface).

However, in addition to this coarse scale phenomenon, we also observe a striking variation of the penetration field for a few disks at very specific locations in the array, as is demonstrated by the images in Fig. 4. In particular, a small proportion of the disks exhibit a positive change in flux at a field much lower than the values of $H_{p}$ plotted in Fig. 2(d). In Fig. 4(a), the change in the flux distribution at $T=45 \mathrm{~K}$ is ini- 
tially strongly modulated by linear channels due to enhanced vortex mobility and density in the brighter areas. Figure 4(b) shows the same region at the slightly higher field of $H_{z}$ $\sim 50$ Oe. Strikingly, disks lying exactly on the border between the high and low flux density regions are now already penetrated even though the applied field is 20 Oe below the usual penetration field (68 Oe) for this temperature. Premature penetration occurs in exactly the same disks over the whole range of investigated temperatures, although at higher temperatures the difference from the average penetration field becomes progressively smaller. It has been demonstrated in Ref. 20 that the vortex density within the linear regions is higher, making it plausible that the local field in the vicinity of the disks in these regions is slightly enhanced. However, in this scenario one would expect all the disks within the defect region to be penetrated earlier and not just those lying at the channel interface. Alternatively we speculate that early penetration is related to a local reduction of the $\mathrm{BLb}$ where the edge of the linear channel intersects the disks, though the exact microscopic origin of this effect remains unclear.

In conclusion, by fabricating arrays of microdisks at the surface of a BSCCO single crystal we have demonstrated that the temperature dependence of the penetration field is well described by the thermal activation of pancake vortices over the Bean-Livingston barrier, with a characteristic temperature $T_{0}=19-25 \mathrm{~K}$. We find that demagnetizing fields are still important in these structures and that the estimated field enhancement at the edge is closest to that for a strip with a rectangular cross section. Finally, we have shown that the penetration field is lower at the edge of linear defects, suggesting that the BLb is suppressed by this form of quenched microscopic crystalline disorder.

This work was supported by EPSRC-U.K. Grant No. GR/ D034264/1 and the Royal Society International Joint Project No. 2006/R3. M.V.M. acknowledges support from MarieCurie Intra-European program. T.T. acknowledges support from JSPS bilateral cooperative program.
${ }^{1}$ C. P. Bean and J. D. Livingston, Phys. Rev. Lett. 12, 14 (1964).

${ }^{2}$ M. Nideröst, R. Frassanito, P. Visani et al., Physica C 282, 1301 (1997).

${ }^{3}$ R. A. Doyle, S. F. W. R. Rycroft, C. D. Dewhurst et al., Physica C 308, 123 (1998).

${ }^{4}$ Y. M. Wang, A. Zettl, S. Ooi, and T. Tamegai, Phys. Rev. B 65, 184506 (2002).

${ }^{5}$ L. N. Bulaevskii, M. Ledvij, and V. G. Kogan, Phys. Rev. B 46, 366 (1992).

${ }^{6}$ L. Burlachkov, Phys. Rev. B 47, 8056 (1993).

${ }^{7}$ V. N. Kopylov, A. E. Koshelev, and I. F. Schegolev, Physica C 170, 291 (1990).

${ }^{8}$ E. Zeldov, A. I. Larkin, V. B. Geshkenbein, M. Konczykowski, D. Majer, B. Khaykovich, V. M. Vinokur, and H. Shtrikman, Phys. Rev. Lett. 73, 1428 (1994).

${ }^{9}$ N. Morozov, E. Zeldov, M. Konczykowski, and R. A. Doyle, Physica C 291, 113 (1997); D. Majer, E. Zeldov, and M. Konczykowski, Phys. Rev. Lett. 75, 1166 (1995).

${ }^{10}$ I.-F. Tsu, J.-L. Wang, S. E. Babcock et al., Physica C 349, 8 (2001).

${ }^{11}$ G. Yang, J. S. Abell, and C. E. Gough, Physica C 341, 1091 (2000); M. Connolly, S. J. Bending, A. N. Grigorenko, and T.
Tamegai, Phys. Rev. B 72, 224504 (2005)

${ }^{12}$ Y. Fasano, M. Menghini, F. de la Cruz, and G. Nieva, Phys. Rev. B 62, 15183 (2000)

${ }^{13}$ M. R. Koblischka, R. J. Wijngaarden, D. G. de Groot et al., Physica C 249, 339 (1995); M. Yasugaki, M. Tokunaga, N. Kameda, and T. Tamegai, Phys. Rev. B 67, 104504 (2003).

${ }^{14}$ M. Li, P. H. Kes, S. F. W. R. Rycroft et al., Physica C 408, 25 (2004).

${ }^{15}$ T. Tamegai, M. Matsui, M. Yasugaki, Y. Tokunaga, and M. Tokunaga, Magneto-Optical Imaging, NATO Science Series B: Physics Vol. 142 (Kluwer, Dordrecht, 2004).

${ }^{16}$ M. S. James, S. T. Stoddart, S. J. Bending, S. Aukkaravittayapun, P. J. King, and M. Henini, Phys. Rev. B 56, R5771 (1997).

${ }^{17}$ E. Zeldov, J. R. Clem, M. McElfresh, and M. Darwin, Phys. Rev. B 49, 9802 (1994).

${ }^{18}$ M. V. Indenbom, C. J. van der Beek, V. Berseth et al., J. Low Temp. Phys. 105, 1529 (1996).

${ }^{19}$ J. A. Herbsommer, V. F. Correa, G. Nieva et al., Solid State Commun. 59, 120 (2001).

${ }^{20}$ A. Oral, J. C. Barnard, S. J. Bending, S. Ooi, H. Taoka, T. Tamegai, and M. Henini, Phys. Rev. B 56, R14295 (1997). 